\title{
Modeling and simulating the BrNo3 Nano sensor for illness Diagnosis
}

\author{
Ali Goli ${ }^{1}$, VahidAzimiRaad ${ }^{2}$, QolamrezaKiani ${ }^{3}$ and Ahmad Qanbari ${ }^{4}$ \\ ${ }^{1}$ Tehran Medical University, Medical School, Medical Physics and Engineering, \\ ${ }^{2}$ Tabriz University, Center of Excellence in Mechatronics, Novel Technology Engineering Faculty, \\ ${ }^{3}$ Tabriz University, Center of Excellence in Nano photonic, Novel Technology Engineering Faculty, \\ ${ }^{4}$ Tabriz University, Center of Excellence in Mechatronics, Novel Technology Engineering Faculty,
}

\begin{abstract}
Using of the BrNo3 Nanotubes is increasing because of their high biologic compatibility. In this study, the Euler-Bernoulli equations have been utilized analytically to improve the solvation speed and diagnosis. Finally, the equation has been solved by the numerical solution method and various modes have been obtained.
\end{abstract}

Keywords: BrNo3 Nanotubes, Frequency change, Cantilevers, Euler-Bernoulli Equation.

\section{Introduction}

Carbon Nanotubes have attracted the considerable researches because of their great electrical, thermal and mechanical properties [1, 2, 3 and 4]. Recently the carbon Nanotube application determined in biologic applications such as biologic sensors and great developments obtained in wide domain [5 and 6]. Many findings such as Nanotube carbon sensors of field effect transistors [7] and Nanotube carbon biosensors of cantilever trick [8]. Blasé [9] predicted the BrNo3 Nanotubes, exploring the carbon Nanotubes. They started to research on individual nanotube's properties [10] and [11]. They also worked on hexagonal BrNo3 and graphite similar properties [12]. Rising and developing a hexagonal BrNo3 plate in different chiral directions of BrNo3 Nanotubes with different properties. They have the most of the upstream and excellent properties [13 and 14] such as especial elastic properties [15 and 16], high mechanic resistivity [17 and 18], chemical inaction [19], structural stability [20 and 21], high heat transmission and piezoelectric properties [22]. Eventually, BrNo3 Nanotubes have a wide space and independent of atomic and geometric structure. These properties satisfy the BrNo3 Nanotubes for the biologic applications [23]. In addition, the BrNo3 Nanotubes is a non-absorbing the visible and invisible light. These properties help to protect the biologic fragments against the heat exhaustion and damage.

Many tries have been done to discover the carbon Nanotubes applications in biotechnology while the BrNo3 Nanotubes applications in wide range. There are some studies about the BrNo3 Nanotubes application as the Nano sensor [24]. Ciofani [20] worked on BrNo3 Nanotubes in Nano drug field. Empirical studies show that BrNo3 Nanotubes are suitable for the sensors, convertors and biologic material determination because of their chemical stability. Ciofani did a study on interactions and the chemical effects on biologic material effects and BrNo3 Nanotubes. In addition, he studied the complexity of BrNo3 Nanotubes. The base theory of Nano sensors founded in frequency transmission [26, 27 and 28] or changing in conductivity [29]. Here they worked on a method for active utilizing of BrNo3 Nanotubes as a biosensor, which recognize the amount of biologic material. In this study, a shift in resonance frequency has been utilized, adding on BrNo3 Nanotubes oscillator $[18,31$ and 32].

Analytical solution of Euler-Bernoulli equation

For the simulation of beam equation or the dynamic modelling of cantilever beam, the Euler-Bernoulli equation should be solved based on initial and boundary conditions. Some variable parameters shown in figure 1.

$E I \frac{\partial^{4} u(x, t)}{\partial x^{4}}+\rho A \frac{\partial^{2} u(x, t)}{\partial t^{2}}=0$ 


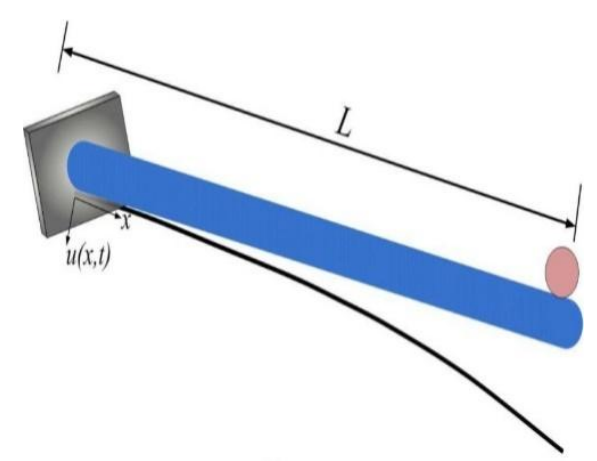

Fig1. Cantilever beam with BrNo3 Nanotubes material in L length and a stick mass at the end

The equation 2 is used for the first equation solving

$$
u(x, t)=\emptyset(x) e^{i \omega t}
$$

$$
E I \varnothing^{4} e^{i \omega t}+\rho A\left(-\omega_{\mathrm{i}}^{2}\right) \emptyset e^{i \omega t}=0
$$

Therefore, we have:

Assuming the $\beta^{4}=\frac{\rho A \omega_{i}^{2}}{E I}$ (4) and its replacing to above equation, we have:

$$
\emptyset^{4}-\beta^{4} \varnothing=0
$$

Considering the equation 5 :

$\omega_{\mathrm{i}}=\sqrt{\frac{\mathrm{EI} \beta^{4}}{\rho A}} \Rightarrow \omega_{\mathrm{i}}=\sqrt{\frac{\mathrm{EI}}{\rho A L^{4}}} \beta^{2} L^{2}$

As it can be seen, the above equation should be solved, having the boundary condition. The boundary condition for the Euler-Bernoulli equations are different considering the beam type. For a one-cantilever beam the below equation has been obtained:

$2+2 \cos \beta L \cosh \beta L=0$

For the equation 7 solvation, the Newton Raphson method should be used. After the solvation, equation will obtain at first frequency mode. The amount at first mode to fifth one is as below:

Figure two shows the graphs belong to upper modes. It can be said that system resonance to oscillation is in especial frequencies that called resonant frequency. In this kind of frequencies, the outer oscillation energy is saved into the object. Due to that, a small and continuous force can originate an oscillation movement with wide domain. In this step, a frequency mode will be generated for each cantilever beam. Figure 2 shows the fifth resonant frequency.

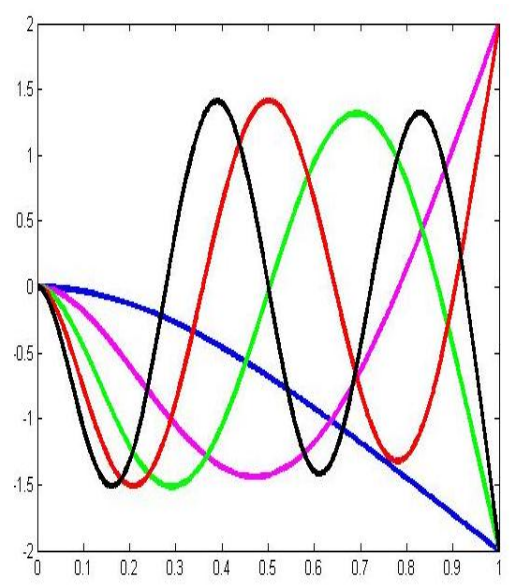

Fig2. Different modes per various resonant frequencies 
$E=2.73 \times 10^{12} p a$

$I=\frac{\pi}{4} r^{4}=4.5246 \times 10^{-39} \mathrm{~m}^{4}$

$\rho=3074.1 \mathrm{~kg} / \mathrm{m}^{3}$

$A=\pi r^{2}=2.3845 \times 10^{-19} \mathrm{~m}^{2}$

$\beta L=1.8751$

$m_{0}=9.9682 \times 10^{-24} \mathrm{~kg}$

Where $\mathrm{L}$ is the length of beam, $\mathrm{E}$ is Young's modulus, I is the second torque of transverse section $\mathrm{A}, \rho$ is the beam density, $\mathrm{A}$ is the cross section surface and $\beta$ is the initial mass. Therefore, we have:

The main $\omega_{\mathrm{i}}$ is obtained, dividing the equation 15 on $2 \pi$ (equation 16 ).

$16\left(\omega_{\mathrm{i}}=\frac{257.07}{2} \pi=40.9140 \mathrm{GHz}\right.$

Now, having an extra mass (x), the $\rho$ changes and it causes the frequency change consequently.

$$
\begin{aligned}
& m=m_{0}+x=(9.9682+0.121307) \times 10^{-24} \\
& \rho=\frac{m}{\mathrm{AL}}=\frac{(9.9682+0.121307) \times 10^{-24}}{2.3845 \times 10^{-19} \times 7 x} \\
& \omega_{\mathrm{i}}=\sqrt{\frac{\mathrm{EI}}{\rho A L\left(L^{3}\right)}} \beta^{2} L^{2}=\sqrt{\frac{\mathrm{EI}}{m_{e q}\left(L^{3}\right)}} \beta^{2} L^{2}
\end{aligned}
$$

Via the equivalent mass (m_eq) and its obtaining by the energy method it can be arrived (equation 18). For Cantilever beam, we have:

Potential Energy:

(20)

$$
\begin{aligned}
& U_{1}(x)=\left(\cosh \beta L \frac{x}{L}-\cos \beta L \frac{x}{L}\right)-\left(\frac{\sinh \beta L-\sin \beta L}{\cosh \beta L+\cos \beta L}\right)\left(\sinh \beta L \frac{x}{L}-\sin \beta L \frac{x}{L}\right) \\
& U(x)=a U_{1}(x) \\
& \beta L=1.8751
\end{aligned}
$$

Integrating the $\mathrm{x}$, finally $\mathrm{P}$ obtained as below:

$$
P=12.36236338 a^{2} \frac{E I}{L^{3}}
$$

The equivalent mass obtained via the below energy:

$P=\frac{1}{2} K_{e q} a^{2} \Rightarrow K_{e q}=12.36236338 \frac{E I}{L^{3}}$

The set mass on beam (M) considered. The frequency is $\omega$ and the Kinetic energy calculated as below:

$$
T=\frac{\omega^{2}}{2} \int_{0}^{l} \rho A U^{2}(x) d x+\frac{\omega^{2}}{2} M U^{2}(L)
$$

Where 1 is the integral limitation, $\mathrm{L}$ is the location of mass (M) at the end of beam. It, length will be calculated, locating in each point.

Since that, we have below equation for energy:

$$
T=\frac{1}{2} m_{e q} \omega^{2} a^{2}
$$


The equivalent mass for a cantilever beam obtained as below:

$m_{e q}=\rho A L+4 M$

Replacing the above equation, we have the below equation:

Now, replacing that equation, a new frequency obtain that it can compared with initial frequency and consequently find the added mass (frequency shift).

\section{Conclusion}

In this article, the various benefits of $\mathrm{BrNo} 3$ Nanotubes have been explained replacing the carbon Nanotubes. A BrNo3 Nanotube modelled using the Euler equation and finally the simulation done by the MATLAB software. Eventually a novel and precise procedure introduced for the added mass on beam. Increasing the determination speed, the numerical-Analytical simulation was used replacing the numerical solution.

\section{Reference}

[1]. T. W. Odom, J. L. Huang, P. Kim, and C. M. Lieber, "Atomic structure and electronic properties of single walled carbon nanotubes," Nature, vol. 391, no. 6662, pp. 62-64, 1998

[2]. B. Babic, J. Furer, S. Sahoo, S. Farhangfar, and C. Schonenberger, "Intrinsic thermal vibrations of suspended doubly clamped single wall carbon nanotubes," NanoLett., vol. 3, no. 11, pp. 1577-1580, 2003

[3]. V. N. Popov, V. E. Van Doren, and M. Balkanski, "Elastic properties of single walled carbon nanotubes," Phys. Rev. B, vol. 61, no. 4, pp. 3078-3084, 2000.

[4]. D. Sanchez Portal, E. Artacho, J. M. Soler, A. Rubio, and P. Ordejon, "Abinitio structural, elastic, and vibrational properties of carbon nanotubes,’Phys. Rev. B, vol. 59, no. 19, pp. 12 678-12 688, 1999.

[5]. R. Singh, D. Pantarotto, L. Lacerda, G. Pastorin, C. Klumpp, M. Prato,A. Bianco, and K.Kostarelos, "Tissuenum bio distribution and blood clearance rates of intravenously administered carbon nanotube radio tracers,” Proc. Natl. Acad. Sci., vol. 103, no. 9, pp. 3357-3362, 2006

[6]. C. Y. Li and T. W. Chou, "Mass detection using carbon nanotube based nanomechanical resonators," Appl. Phys. Lett., vol. 84, no. 25, pp. 52465248, 2004

[7]. B. L. Allen, P. D. Kichambare, and A. Star, "Carbon nanotube field effecttransistor based biosensors," Adv. Mater., vol. 19, no. 11, pp. 1439-1451, 2007

[8]. K. Jensen, K. Kim, and A. Zettl, “An atomic resolution nanomechanical mass sensor,” Nature Nanotechnol., vol. 3, no. 9, pp. 533537,2008

[9]. X. Blase, A. Rubio, S. Louie, and M. L. Cohen, "Stability and band gap constancy of boron nitride nanotubes," EurophysicsLett., vol. 28, no. 5,pp. 335-340, 1994.

[10]. N. Chopra, R. Luyken, K. Cherrey, V. Crespi, M. Cohen, S. Louie, and A. Zettl, "Boron nitride nanotubes," Science, vol. 269, no. 5226, pp. $966967,1995$.

[11]. D. Golberg, Y. Bando, C. Tang, and C. Zhi, "Boron nitride nanotubes,"Adv. Mater., vol. 19, no. 18, pp. $2413-2432,2007$.

[12]. C. Y.Won and N. R. Aluru, "Structure and dynamics of water confined in a boron nitride nanotube," J. Phys. Chem. C, vol. 112, no. 6, pp. 1812-1818,2008.

[13]. M. Santosh, P. K. Maiti, and A. K. Sood, "Elastic properties of boron nitride nanotubes and their comparison with carbon nanotubes,” J. Nanosci.Nanotechnol., vol. 9, no. 9, pp. 5425-5430, 2009.

[14]. J. Yuan and K.M. Liew, "Effects of boron nitride impurities on the elastic properties of carbon nanotubes," Nanotechnology, vol. 19, no. 44, 2008.

[15]. F. Xu, Y. Bando, D. Golberg, R. Ma, Y. Li, and C. Tang, "Elastic deformation of helical conical boron nitride nanotubes," J. Chem. Phys., vol. 119, no. 6, pp. 3436-3440, 2003

[16]. L. Wirtz, A. Rubio, R. A. de la Concha, and A. Loiseau, "Ab initio calculations of the lattice dynamics of boron nitride nanotubes," Phys. Rev. B, vol. 68, no. 4, p. 045425, 2003.

[17]. Q. Huang, Y. Bando, X. Xu, T. Nishimura, C. Zhi, C. Tang, F. Xu, L. Gao, and D. Golberg, "Enhancing superplasticity of engineering ceramics by introducing BN nanotubes," Nanotechnology, vol. 18, no. 48, 2007.

[18]. G. S. Jeon and G. D. Mahan, "Lattice vibrations of a single wall boron nitride nanotube,” Phys. Rev. B, vol. 79, no. 8, 2009.

[19]. C. Y. Zhi, Y. Bando, C. C. Tang, Q. Huang, and D. Golberg, "Boron nitride nanotubes: Functionalization and composites," J. Mater. Chem., vol. 18, no. 33, pp. 3900-3908, 2008.

[20]. D. Golberg, Y. Bando, K. Kurashima, and T. Sato, "Synthesis and characterization of ropes made of BN multiwalled nanotubes," Scr. Mater., vol. 44, no. 8-9, pp. 1561-1565, 2001.

[21]. G. Ciofani, V. Raffa, A. Menciassi, and A. Cuschieri, "Boron nitride nanotubes: An innovative tool for nanomedicine," Nano Today, vol. 4, no. 1, pp. 8-10, 2009.

[22]. E. S. Oh, "Elastic properties of boron nitride nanotubes through the continuum lattice approach," Mater. Lett., vol. 64, no. 7, pp. 859-862, 2010.

[23]. C. Zhi, Y. Bando, C. Tang, and D. Golberg, "Immobilization of proteins on boron nitride nanotubes,” J. Am. Chem. Soc., vol. 127, no. 49, pp. 1714417 145, 2005.

[24]. G. Ciofani, V. Raffa, A. Menciassi, and P. Dario, "Preparation of boron nitride nanotubes aqueous dispersions for biological applications," J.Nanosci. Nanotechnol., vol. 8, no. 12, pp. 6223-6231, 2008

[25]. G. Ciofani, S. Danti, D. D’Alessandro, S. Moscato, and A. Menciassi, "Assessing cytotoxicity of boron nitride nanotubes: Interference with the MTT assay,” Biochem. Biophys. Res. Commun., vol. 394, no. 2, pp. 405-411, 2010. 
[26]. K. Jensen, K. Kim, and A. Zettl, “An atomic resolution nanomechanical mass sensor,” Nature Nanotechnol., vol. 3, no. 9, pp. 533$537,2008$.

[27]. S. K. Georgantzinos and N. K. Anifantis, "Carbon nanotube based resonant nanomechanical sensors: A computational investigation of their behavior,” Phys. E: Low Dimensional Sys. Nanostruct., vol. 42, no. 5,pp. 1795-1801,2010.

[28]. S. Adhikari and R. Chowdhury, "The calibration of carbon nanotube based bio nano sensors,” J. Appl. Phys., vol. 107, no. 12, pp. $124322: 1-8,2010$.

[29]. L. C. Jiang and W. D. Zhang, "A highly sensitive nonenzymatic glucose sensor based on cuo nanoparticles modified carbon nanotube electrode,"Biosens. Bioelectron., vol. 25, no. 6, pp. 1402-1407, 2010.

[30]. S. B. Tooski, "Functionalized single wall carbon nanotube sensor in a perturbed microwave resonant cavity based toxin/pollutant gas pressure sensor," J. Appl. Phys., vol. 107, no. 3, 2010.

[31]. E. Hern'andez, C. Goze, P. Bernier, and A. Rubio, "Elastic properties of c and bxcynzcomposite nanotubes," Phys. Rev. Lett., vol. 80, no. 20, pp. 4502-4505, 1998.

[32]. D. S'anchez Portal and E. Hern'andez, "Vibrational properties of singlewall nanotubes and monolayers of hexagonal BN," Phys. Rev. B, vol. 66, no. 23, p. 235415, 2002. 